\title{
Estimates for the Norm of Generalized Maximal Operator on Strong Product of Graphs
}

\author{
Zaryab Hussain $\mathbb{D D}^{1,2}$ Ghulam Murtaza $\mathbb{D D}^{3},{ }^{3}$ Toqeer Mahmood $\mathbb{D}^{4},{ }^{4}$ and Jia-Bao Liu $\mathbb{D}^{5}$ \\ ${ }^{1}$ School of Mathematics and Statistics, Northwestern Polytechnical University, Xi'an, Shaanxi 710129, China \\ ${ }^{2}$ Department of Mathematics, Government College University, Faisalabad 38000, Pakistan \\ ${ }^{3}$ Department of Mathematics, School of Science, University of Management and Technology, Lahore 54000, Pakistan \\ ${ }^{4}$ Department of Computer Science, National Textile University, Faisalabad 37610, Pakistan \\ ${ }^{5}$ School of Mathematics and Physics, Anhui Jianzhu University, Hefei 230601, China \\ Correspondence should be addressed to Toqeer Mahmood; toqeer.mahmood@yahoo.com
}

Received 20 April 2021; Accepted 11 June 2021; Published 9 July 2021

Academic Editor: Sakander Hayat

Copyright (C) 2021 Zaryab Hussain et al. This is an open access article distributed under the Creative Commons Attribution License, which permits unrestricted use, distribution, and reproduction in any medium, provided the original work is properly cited.

Let $G=G_{1} \times G_{2} \times \cdots \times G_{m}$ be the strong product of simple, finite connected graphs, and let $\phi: \mathbb{N} \longrightarrow(0, \infty)$ be an increasing function. We consider the action of generalized maximal operator $M_{\mathrm{G}}^{\phi}$ on $\ell^{p}$ spaces. We determine the exact value of $\ell^{p}$-quasinorm of $M_{G}^{\phi}$ for the case when $G$ is strong product of complete graphs, where $0<p \leq 1$. However, lower and upper bounds of $\ell^{p}$-norm have been determined when $1<p<\infty$. Finally, we computed the lower and upper bounds of $\left\|M_{G}^{\phi}\right\|_{p}$ when $G$ is strong product of arbitrary graphs, where $0<p \leq 1$.

\section{Introduction}

We review some of the standard facts on graphs and metric on the graphs. All the graphs considered in this paper are simple, finite, and connected. Let $G(V(G), E(G))$ be a graph, where $V(G)$ is the set of vertices and $E(G)$ is the set of edges of $G$. The vertices which are at distance one from any vertex $x \in V(G)$ are called neighbors of $x$. The set of neighbors of $x \in V(G)$ is denoted by $N_{G}(x)$. The degree of any vertex $x \in V(G)$ is the cardinality of the set $N_{G}(x)$ and is denoted by $d_{G}(x)$. The distance between two vertices $x$ and $y$ denoted by $d(x, y)$ is the length of the shortest path between $x$ and $y$. For more details on graph theory, we refer the readers to [1-3]. The metric (graph metric) $d_{G}: V(G) \times V(G) \longrightarrow \mathbb{R}$ on graph $G$ is defined as

$$
d_{G}(x, y)=\text { distance between } x \text { and } y,
$$

where $x, y \in V(G)$. This metric space $\left(G, d_{G}\right)$ is called geodesic metric space. For any function $f: V(G) \longrightarrow \mathbb{R}$, the
Hardy-Littlewood maximal operator $M_{G}^{x}: \ell^{p} \longrightarrow \ell^{p}[4-7]$ is defined as

$$
M_{G}^{x} f(q)=\sup _{r \geq 0} \frac{1}{|B(q, r)|} \sum_{w \in B(q, r)}|f(w)|,
$$

where $B(q, r)=\left\{s \in V(G): d_{G}(q, s) \leq r\right\}$ is the ball with center $q \in V(G)$ and radius $r$ on a graph $G$. It contains all the vertices of $G$ which are at distance atmost $r$ from the vertex $q$. It is clear from the definition that if $r=0$, then $|B(q, r)|=1$, and if $r \geq 1$, then $|B(q, r)| \geq 2$. The values of metric function $d_{G}$ are natural numbers and radius $r \geq 0$; therefore, equation (2) can be written as

$$
M_{G}^{x} f(q)=\max _{r \in \mathbb{N}} \frac{1}{|B(q, r)|} \sum_{w \in B(q, r)}|f(w)| .
$$

The fractional maximal operator [8] on graphs is defined as 


$$
M_{G}^{1-(t / n)} f(q)=\max _{r \in \mathbb{N}} \frac{1}{|B(q, r)|^{1-(t / n)}} \sum_{w \in B(q, r)}|f(w)|,
$$

where $0 \leq t \leq n$. If $t=0$, then equation (4) reduces to equation (3). For $0<p<\infty$, the $\ell^{p}$ norm of the Hardy-Littlewood maximal operator is defined as

$$
\left\|M_{G}^{x}\right\|_{\ell^{p}}:=\sup _{f \neq 0} \frac{\left\|M_{G}^{x} f\right\|_{\ell^{p}}}{\|f\|_{\ell^{p}}}
$$

where $\|f\|_{\ell^{p}}=\left(\sum_{s \in V(G)}|f(s)|^{p}\right)^{(1 / p)}$.

For every function $f: V(G) \longrightarrow \mathbb{R}$, the generalized maximal operator $M_{G}^{\phi}: \ell^{p} \longrightarrow \ell^{p}[9,10]$ is defined as

$$
M_{G}^{\phi} f(q)=\max _{r \in \mathbb{N}} \frac{1}{\phi(|B(q, r)|)} \sum_{w \in B(q, r)}|f(w)|,
$$

where $\phi: \mathbb{N} \longrightarrow(0, \infty)$ is an increasing function. Note that if we take $\phi(x)=x$ in equation (6), then we get the classical Hardy-Littlewood maximal operator $M_{G}^{x}$, and if we take $\phi(x)=x^{1-(t / n)}$ in equation (5), then we get equation (4).

Let $K_{n}$ be complete graph on $n$ vertices. For any vertex $q \in V\left(K_{n}\right)$, the ball $B(q, r)$ with center $q$ and radius $r$ is defined as

$$
B(q, r)= \begin{cases}\{q\}, & \text { for } r=0, \\ V\left(K_{n}\right), & \text { for } r \geq 1\end{cases}
$$

Therefore, the generalized maximal operator on complete graph $K_{n}$ takes the form

$$
M_{K_{n}}^{\phi} f(q)=\max \left\{\frac{1}{\phi(1)}|f(q)|, \frac{1}{\phi(n)} \sum_{v \in V\left(K_{n}\right)}|f(v)|\right\} .
$$

For any vertex $i \in V(G)$, the Kronecker delta function is defined as

$$
\delta_{q}(i)= \begin{cases}1, & q=i, \\ 0, & q \neq i .\end{cases}
$$

Soria and Tradacete [6] estimated the norm of maximal operator $M_{G}^{x}$ in the following form.
Proposition 1 (see [6])

(i) If $0<P \leq 1$, then

$$
\left\|M_{K_{n}}\right\|_{p}=\left(1+\frac{n-1}{n^{p}}\right)^{(1 / p)} .
$$

(ii) If $1<p<\infty$, then

$$
\left(1+\frac{n-1}{n^{p}}\right)^{(1 / p)} \leq\left\|M_{K_{n}}\right\|_{p} \leq\left(1+\frac{n-1}{n}\right)^{(1 / p)} .
$$

For more details on this topic of research, see $[4,8,10-13]$. The main motivation of this paper is from $[4-7,10]$.

The paper is structured as follows. Section 2 contains the definitions which are helpful to prove the main results. Section 3 contains the main results; we find the exact value of $\left\|M_{\mathbf{K}}^{\phi}\right\|_{p}$ for the case $0<p \leq 1$ and give lower and upper bound when $1<p<\infty$. An example is given to show that these bounds are not optimal. Finally, Section 4 concludes the study.

\section{Preliminaries}

Let $G_{1}, G_{2}, \ldots, G_{m}$ be $m$ graphs; then, their strong product $G=G_{1} \times G_{2} \times \cdots \times G_{m}$ is a graph having vertex set,

$$
V(G)=\left\{\left(u_{1}, u_{2}, \ldots, u_{m}\right): u_{i} \in G_{i} \forall i=1,2, \ldots, m\right\},
$$

and the edge set, which is defined in the following manner; there will be an edge between $\left(u_{1}, u_{2}, \ldots, u_{m}\right)$ and $\left(v_{1}, v_{2}, \ldots, v_{m}\right)$ in $G$ if
(a) $u_{i}=v_{i}$ and $\left(u_{j}, v_{j}\right) \in E\left(G_{j}\right), j \neq i$
(b) $u_{i} \neq v_{i}$ and $\left(u_{i}, v_{i}\right) \in E\left(G_{i}\right), \forall i$

Example 1. Let $K_{2}$ be complete graph on two vertices. The strong product $K=K_{2} \times K_{2} \times K_{2}$ of three $K_{2}$ graphs is shown in Figure 1.

Let $G$ be the strong product of $m$ graphs. Then, for every function $f: V(G) \longrightarrow \mathbb{R}$, we can consider the generalized maximal operator $M_{G}^{\phi}: \ell^{p} \longrightarrow \ell^{p}$ as

$$
M_{G}^{\phi} f\left(u_{1}, u_{2}, \ldots, u_{m}\right)=\max _{r \in \mathbb{N}} \frac{\sum_{v_{1} \in G_{1}} \sum_{v_{2} \in G_{2}} \ldots \sum_{v_{m} \in G_{m}}\left|f\left(v_{1}, v_{2}, \ldots, v_{m}\right)\right|}{\phi\left(\left|B_{G_{1}}\right| \times\left|B_{G_{2}}\right| \times \cdots \times\left|B_{G_{m}}\right|\right)},
$$

where $B_{G_{i}}=B\left(u_{i}, r\right), i=1,2, \ldots, m$. The norm $\left\|M_{G}^{\phi}\right\|_{p}$ of the generalized maximal operator is defined as

$$
\left\|M_{G}^{\phi}\right\|_{p}=\sup _{f \neq 0} \frac{\left\|M_{G}^{\phi} f\right\|_{p}}{\|f\|_{p}}
$$

where $\|f\|_{p}=\left(\sum_{v_{1} \in G_{1}} \sum_{v_{2} \in G_{2}} \ldots \sum_{v_{m} \in G_{m}} \mid f\left(v_{1}, v_{2}, \ldots\right.\right.$, $\left.\left.v_{m}\right)\left.\right|^{p}\right)^{(1 / p)}$.

Let $K=K_{n_{1}} \times K_{n_{2}} \times \cdots \times K_{n_{m}}$ be the strong product of $m$ complete graphs with $n_{1}, n_{2}, \ldots, n_{m}$ vertices, respectively; then, 


$$
B\left(u_{1}, r\right) \times B\left(u_{2}, r\right) \times \cdots \times B\left(u_{m}, r\right)= \begin{cases}\left\{\left(u_{1}, u_{2}, \ldots, u_{m}\right)\right\}, & \text { for } r=0 \\ V(K), & \text { for } r \geq 1\end{cases}
$$

For every function $f: V(K) \longrightarrow \mathbb{R}$, the generalized maximal operator takes the form

$$
M_{\mathbf{K}}^{\phi} f\left(u_{1}, u_{2}, \ldots, u_{m}\right)=\max \left\{\begin{array}{c}
\frac{1}{\phi(1)}\left|f\left(u_{1}, u_{2}, \ldots, u_{m}\right)\right| \\
\frac{1}{\phi\left(n_{1} \times n_{2} \times \cdots \times n_{m}\right)} \sum_{v_{1} \in K_{n_{1}}} \sum_{v_{2} \in K_{n_{2}}} \ldots \sum_{v_{m} \in K_{n_{m}}}\left|f\left(v_{1}, v_{2}, \ldots, v_{m}\right)\right|
\end{array}\right\} .
$$

Note that the operator $M_{K}^{\phi}$ is the smallest in the pointwise ordering among all $M_{G=G_{1} \times G_{2} \times \cdots \times G_{m}}^{\phi}$, where each $G_{i}$ is a graph with $n_{i}$ vertices for $i=1,2, \ldots, m$. That is, for every nonnegative function $f$ and every vertex $\left(u_{1}, u_{2}, \ldots, u_{m}\right) \in G$, we have that

$$
M_{K}^{\phi} f\left(u_{1}, u_{2}, \ldots, u_{m}\right) \leq M_{G}^{\phi} f\left(u_{1}, u_{2}, \ldots, u_{m}\right) .
$$

In particular, if $0<p<\infty$, then

$$
\left\|M_{K}^{\phi}\right\|_{p}^{p} \leq\left\|M_{G}^{\phi}\right\|_{p}^{p}
$$

For any vertex $\left(u_{1}, u_{2}, \ldots, u_{m}\right) \in V(K)$, the $m$ Dirac delta function is defined as

$$
\Gamma_{\left(u_{1}, u_{2}, \ldots, u_{m}\right)}\left(v_{1}, v_{2}, \ldots, v_{m}\right)=\delta_{u_{1}}\left(v_{1}\right) \cdot \delta_{u_{2}}\left(v_{2}\right) \ldots \delta_{u_{m}}\left(v_{m}\right) .
$$

It is easy to check that

$\Gamma_{\left(u_{1}, u_{2}, \ldots, u_{m}\right)}\left(v_{1}, v_{2}, \ldots, v_{m}\right)= \begin{cases}0, & \text { for } u_{j} \neq v_{j}, \text { for some } j, \\ 1, & \text { for } u_{i}=v_{i}, \forall i=1,2, \ldots, m .\end{cases}$

\section{Main Results}

This section details the steps to find the quasi-norm of $M_{K}^{\phi}$, for the case $0<p \leq 1$, and to find bounds of $\left\|M_{K}^{\phi}\right\|_{p}$, for the case of $1<p<\infty$. Also, we estimate the bounds of $\left\|M_{G}^{\phi}\right\|_{p}$ for $0<p \leq 1$. Moreover, some examples are presented to support the results.

Lemma 1. Let $G$ be the strong product of $m$ graphs, and $\Omega: \ell^{p} \longrightarrow \ell^{p}$ be a sublinear operator, with $0<p \leq 1$. Then,

$$
\|\Omega\|_{p}=\max _{\left(u_{1}, u_{2}, \ldots, u_{m}\right) \in V(G)}\left\|\Omega \Gamma_{\left(u_{1}, u_{2}, \ldots, u_{m}\right)}\right\|_{p} .
$$

Proof. Since $\left\|\Gamma_{\left(u_{1}, u_{2}, \ldots, u_{m}\right)}\right\|_{p}=1$, therefore $\|\Omega\|_{p} \geq$ $\max _{\left(u_{1}, u_{2}, \ldots, u_{m}\right) \in V(\mathbf{G})}\left\|\Omega \Gamma_{\left(u_{1}, u_{2}, \ldots, u_{m}\right)}\right\|_{p}$. To prove the other inequality, let $h: V(G) \longrightarrow \mathbb{R}$, with $\|h\|_{p} \leq 1$, that is,

$$
h=\sum_{u_{1} \in G_{1}} \sum_{u_{2} \in G_{2}} \ldots \sum_{u_{m} \in G_{m}} a_{\left(u_{1}, u_{2}, \ldots, u_{m}\right)} \Gamma_{\left(u_{1}, u_{2}, \ldots, u_{m}\right)},
$$

with $\quad \sum_{u_{1} \in G_{1}} \sum_{u_{2} \in G_{2}} \ldots \sum_{u_{m} \in G_{m}}\left|a_{\left(u_{1}, u_{2}, \ldots, u_{m}\right)}\right|^{p} \leq 1$. Using Hölder's inequality for $0<p \leq 1$, it follows that

$$
\begin{aligned}
\|\Omega h\|_{p}^{p} & =\sum_{v_{1} \in G_{1}} \sum_{v_{2} \in G_{2}} \ldots \sum_{v_{m} \in G_{m}}\left|\Omega h\left(v_{1}, v_{2}, \ldots, v_{m}\right)\right|^{p} \\
& =\sum_{v_{1} \in G_{1}} \sum_{v_{2} \in G_{2}} \ldots \sum_{v_{m} \in G_{m}}\left|\Omega\left(\sum_{u_{1} \in G_{1}} \sum_{u_{2} \in G_{2}} \ldots \sum_{u_{m} \in G_{m}} a_{\left(u_{1}, u_{2}, \ldots, u_{m}\right)} \Gamma_{\left(u_{1}, u_{2}, \ldots, u_{m}\right)}\left(v_{1}, v_{2}, \ldots, v_{m}\right)\right)\right|^{p} \\
& \leq \sum_{v_{1} \in G_{1}} \sum_{v_{2} \in G_{2}} \ldots \sum_{v_{m} \in G_{m}}\left|\sum_{u_{1} \in G_{1} u_{2} \in G_{2}} \ldots \sum_{u_{m} \in G_{m}}\right| a_{\left(u_{1}, u_{2}, \ldots, u_{m}\right)}\left|\Omega \Gamma_{\left(u_{1}, u_{2}, \ldots, u_{m}\right)}\left(v_{1}, v_{2}, \ldots, v_{m}\right)\right|^{p} \\
& \leq \sum_{v_{1} \in G_{1}} \sum_{v_{2} \in G_{2}} \ldots \sum_{v_{m} \in G_{m}} \sum_{u_{1} \in G_{1}} \sum_{u_{2} \in G_{2}} \ldots \sum_{u_{m} \in G_{m}}\left|a_{\left(u_{1}, u_{2}, \ldots, u_{m}\right)} \Omega \Gamma_{\left(u_{1}, u_{2}, \ldots, u_{m}\right)}\left(v_{1}, v_{2}, \ldots, v_{m}\right)\right|^{p} \\
& =\sum_{u_{1} \in G_{1}} \sum_{u_{2} \in G_{2}} \ldots \sum_{u_{m} \in G_{m}}\left|a_{\left(u_{1}, u_{2}, \ldots, u_{m}\right)}\right|^{p} \sum_{v_{1} \in G_{1}} \sum_{v_{2} \in G_{2}} \ldots \sum_{v_{m} \in G_{m}}\left|\Omega \Gamma_{\left(u_{1}, u_{2}, \ldots, u_{m}\right)}\left(v_{1}, v_{2}, \ldots, v_{m}\right)\right|^{p} \\
& =\sum_{u_{1} \in G_{1}} \sum_{u_{2} \in G_{2}} \ldots \sum_{u_{m} \in G_{m}}\left|a_{\left(u_{1}, u_{2}, \ldots, u_{m}\right)}\right|^{p}\left\|\Omega \Gamma_{\left(u_{1}, u_{2}, \ldots, u_{m}\right)}\right\|_{p}^{p} \\
& \leq \max _{\left(u_{1}, u_{2}, \ldots, u_{m}\right) \in V(G)}\left\|\Omega \Gamma_{\left(u_{1}, u_{2}, \ldots, u_{m}\right)}\right\|_{p}^{p}
\end{aligned}
$$




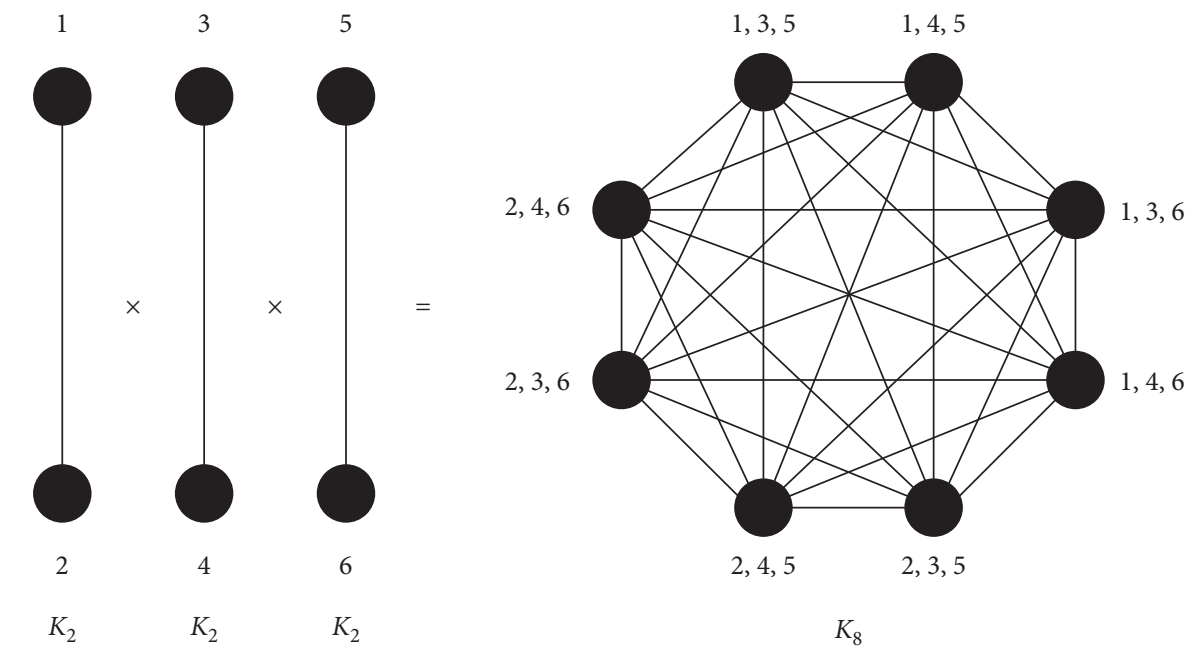

Figure 1: Strong product of three $K_{2}$ graphs.

It completes the proof.

and if $1<p<\infty$, then

Theorem 1. If $0<p \leq 1$, then

$$
\left\|M_{K}^{\phi}\right\|_{p}=\left(\frac{1}{\phi^{p}(1)}+\frac{\left(n_{1} \times n_{2} \times \cdots \times n_{m}\right)-1}{\phi^{p}\left(n_{1} \times n_{2} \times \cdots \times n_{m}\right)}\right)^{(1 / p)},
$$

$$
\begin{aligned}
& \left(\frac{1}{\phi^{p}(1)}+\frac{\left(n_{1} \times n_{2} \times \cdots \times n_{m}\right)-1}{\phi^{p}\left(n_{1} \times n_{2} \times \cdots \times n_{m}\right)}\right)^{(1 / p)} \\
& \quad \leq\left\|M_{K}^{\phi}\right\|_{p} \leq \max \left(\frac{\left(n_{1} \times n_{2} \times \cdots \times n_{m}\right)^{p}}{\phi^{p}\left(n_{1} \times n_{2} \times \cdots \times n_{m}\right)}\right. \\
& \left.\left\{\frac{1}{\phi^{p}(1)}+\frac{\left(\left(n_{1} \times n_{2} \times \cdots \times n_{m}\right)-1\right)\left(n_{1} \times n_{2} \times \cdots \times n_{m}\right)^{(p-1)}}{\phi^{p}\left(n_{1} \times n_{2} \times \cdots \times n_{m}\right)}\right\}\right)^{(1 / p)}
\end{aligned}
$$

Proof. Let $f: V(K) \longrightarrow \mathbb{R}$ be a function such that $\|f\|_{p}=1$. Define $m$ Dirac delta function $\Gamma_{\left(u_{1}, u_{2}, \ldots, u_{m}\right)}$, where $u_{1} \in V\left(K_{n_{1}}\right), u_{2} \in V\left(K_{n_{2}}\right), \ldots, u_{m} \in V\left(K_{n_{m}}\right)$. Then, for $0<p<\infty$, we have

$$
\begin{aligned}
\left\|M_{K}^{\phi} \Gamma_{\left(u_{1}, u_{2}, \ldots, u_{m}\right)}\right\|_{p} & =\left(\left(M_{K}^{\phi} \Gamma_{\left(u_{1}, u_{2}, \ldots, u_{m}\right)}\left(u_{1}, u_{2}, \ldots, u_{m}\right)\right)^{P}+\sum_{v_{1} \neq u_{1}} \sum_{v_{2} \neq u_{2}} \ldots \sum_{v_{m} \neq u_{m}}\left(M_{K}^{\phi} \Gamma_{\left(u_{1}, u_{2}, \ldots, u_{m}\right)}\left(v_{1}, v_{2}, \ldots, v_{m}\right)\right)^{p}\right)^{(1 / p)} \\
& =\left(\frac{1}{\phi^{p}(1)}+\frac{\left(n_{1} \times n_{2} \times \cdots \times n_{m}\right)-1}{\phi^{p}\left(n_{1} \times n_{2} \times \cdots \times n_{m}\right)}\right)^{(1 / p)} .
\end{aligned}
$$

As $\|\Gamma\|_{p}=1$, so we have, for $0<p<\infty$,

$\left(\frac{1}{\phi^{p}(1)}+\frac{\left(n_{1} \times n_{2} \times \cdots \times n_{m}\right)-1}{\phi^{p}\left(n_{1} \times n_{2} \times \cdots \times n_{m}\right)}\right)^{(1 / p)} \leq\left\|M_{K}^{\phi}\right\|_{p}$.
For $0<p \leq 1$, using Lemma 1 , we have

$\left(\frac{1}{\phi^{p}(1)}+\frac{\left(n_{1} \times n_{2} \times \cdots \times n_{m}\right)-1}{\phi^{p}\left(n_{1} \times n_{2} \times \cdots \times n_{m}\right)}\right)^{(1 / p)}=\left\|M_{K}^{\phi}\right\|_{p}$. 
Now, we will prove upper bound for $1<p<\infty$ :

$$
\begin{aligned}
\left\|M_{K}^{\phi} f\right\|_{p}= & \left(\sum _ { u _ { 1 } \in B _ { G _ { 1 } } } \sum _ { u _ { 2 } \in B _ { G _ { 2 } } } \cdots \sum _ { u _ { m } \in B _ { G _ { m } } } \operatorname { m a x } \left\{\frac{1}{\phi(1)}\left|f\left(u_{1}, u_{2}, \ldots, u_{m}\right)\right|,\right.\right. \\
& \left.\left.\cdot \frac{1}{\phi\left(n_{1} \times n_{2} \times \cdots \times n_{m}\right)} \sum_{v_{1} \in B_{G_{1}}} \sum_{v_{2} \in B_{G_{2}}} \ldots \sum_{v_{m} \in B_{G_{m}}}\left|f\left(v_{1}, v_{2}, \ldots, v_{m}\right)\right|^{p}\right\}\right)^{(1 / p)} .
\end{aligned}
$$

After applying Hölder's inequality, we have

$$
\left\|M_{K}^{\phi}\right\|_{p} \leq \sup \left(\sum_{u_{1} \in B_{G_{1}}} \sum_{u_{2} \in B_{G_{2}}} \cdots \sum_{u_{m} \in B_{G_{m}}} \max \left\{\frac{1}{\phi^{p}(1)}\left|f\left(u_{1}, u_{2}, \ldots, u_{m}\right)\right|^{p}, \frac{1}{\phi^{p}\left(n_{1} \times n_{2} \times \cdots \times n_{m}\right)}\left(n_{1} \times n_{2} \times \cdots \times n_{m}\right)^{(p-1)}\right\}\right)^{(1 / p)} .
$$

If $\quad\left(\left|f\left(u_{1}, u_{2}, \ldots, u_{m}\right)\right|^{p} / \phi^{p}(1)\right) \leq\left(\left(n_{1} \times n_{2} \times\right.\right.$ $\left.\left.\cdots \times n_{m}\right)^{(p-1)} / \phi^{p}\left(n_{1} \times n_{2} \times \cdots \times n_{m}\right)\right)$ for all vertices, then we have

$$
\left\|M_{K}^{\phi}\right\|_{p} \leq\left(\frac{\left(n_{1} \times n_{2} \times \cdots \times n_{m}\right)^{p}}{\phi^{p}\left(n_{1} \times n_{2} \times \cdots \times n_{m}\right)}\right)^{(1 / p)}
$$

$$
\begin{aligned}
\left\|M_{K}^{\phi}\right\|_{p} & \leq\left(\frac{1}{\phi^{p}(1)}\left|f\left(u_{1}^{\prime}, u_{2}^{\prime}, \ldots, u_{m}^{\prime}\right)\right|^{p}+\frac{\left(\left(n_{1} \times n_{2} \times \cdots \times n_{m}\right)-1\right)\left(n_{1} \times n_{2} \times \cdots \times n_{m}\right)^{p-1}}{\phi^{p}\left(n_{1} \times n_{2} \times \cdots \times n_{m}\right)}\right)^{(1 / p)} \\
& \leq\left(\frac{1}{\phi^{p}(1)}+\frac{\left(\left(n_{1} \times n_{2} \times \cdots \times n_{m}\right)-1\right)\left(n_{1} \times n_{2} \times \cdots \times n_{m}\right)^{(p-1)}}{\phi^{p}\left(n_{1} \times n_{2} \times \cdots \times n_{m}\right)}\right)^{(1 / p)}
\end{aligned}
$$

which completes our arguments.

The graph of the result of Theorem 1 is shown in Figure 2 , where $\phi(x)=x, n_{1} \times n_{2} \times \cdots \times n_{m}$ is from 4 to 10 , and $p=2$ and $p=3$.

3D solution region for Theorem 1 is shown in Figure 3, where $\phi(x)=x, n_{1} \times n_{2} \times \cdots \times n_{m}$ is from 4 to 12 , and $p$ is from 1 to 10 .

The graph presented in Figure 3 shows the results of Theorem 1 that are not optimal. It is quite difficult task to calculate the exact value of $\left\|M_{K}^{\phi}\right\|_{p}$ for the case $1<p<\infty$. The following example explains the situation.

Example 2. The estimates we obtained in Theorem 1 for $1<$ $p<\infty$ is not optimal in general. For example, if we take graph $K_{2} \times K_{2}$ and $\phi(x)=x$. Consider the function $f:\{(1,3)$, $(1,4),(2,3),(2,4)\} \longrightarrow \mathbb{R}$. We suppose that $|f(1,3)|=\mid f$ $(1,2)|=| f(2,3) \mid, \quad M_{K_{2} \times K_{2}}^{x} f(1,3)=M_{K_{2} \times K_{2}}^{x} f(1,4)=M_{K_{2} \times K_{2}}^{x}$ $f(2,3)=(3|f(1,3)|+|f(1,4)| / 4)$, and $M_{K_{2} \times K_{2}}^{x} f(2,4)=\mid f$
If $\left(\left|f\left(u_{1}^{\prime}, u_{2}^{\prime}, \ldots, u_{m}^{\prime}\right)\right|^{p} / \phi^{p}(1)\right)>\left(\left(n_{1} \times n_{2} \times \cdots \times n_{m}\right)\right)^{(p-1)} /$ $\left.\phi^{p}\left(n_{1} \times n_{2} \times \cdots \times n_{m}\right)\right)$ for some $\left(u_{1}^{\prime}, u_{2}^{\prime}, \ldots, u_{m}^{\prime}\right) \in V(K)$, then we have
$(2,4) \mid$. Then, $|f(2,4)| \geq|f(1,3)|$. If we denote $(|f(1,3)| / \mid f$ $(2,4) \mid)$ by $\lambda$, then, for every $0<p<\infty$, we have

$$
\begin{aligned}
\frac{\left\|M_{K_{2} \times K_{2}}^{x} f\right\|_{p}}{\|f\|_{p}} & =\left(\frac{3(3|f(1,3)|+|f(1,4)| / 4)^{p}+|f(1,4)|^{p}}{3|f(1,3)|^{p}+|f(1,4)|^{p}}\right)^{(1 / p)} \\
& =\frac{1}{4}\left(\frac{3(3 \lambda+1)^{p}+4^{p}}{3 \lambda^{p}+1}\right)^{(1 / p)},
\end{aligned}
$$

which leads to

$$
\left\|M_{K_{2} \times K_{2}}^{x}\right\|_{p}=\frac{1}{4}\left(\sup _{0 \leq \lambda \leq 1} \frac{3(3 \lambda+1)^{p}+4^{p}}{3 \lambda^{p}+1}\right)^{(1 / p)}
$$

It is easy to see that, for $1<p<\infty$, the supremum is attained at the unique root $\lambda_{p} \in(0,1)$ of the equation 


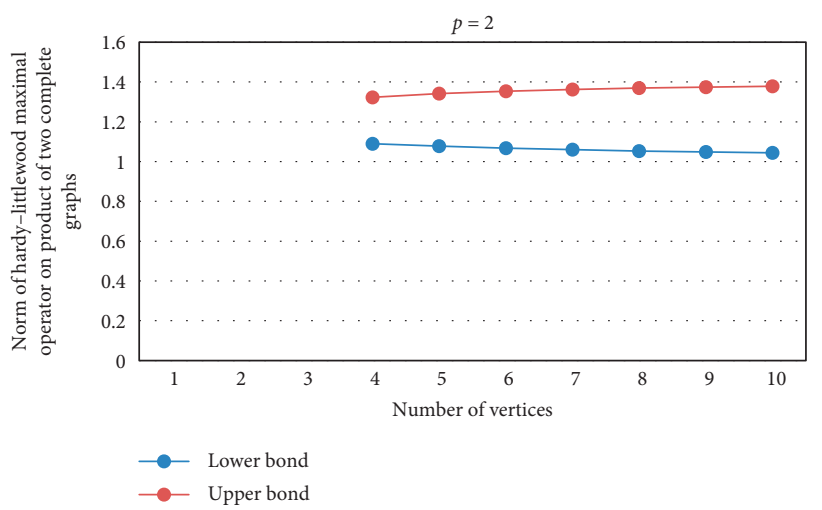

(a)

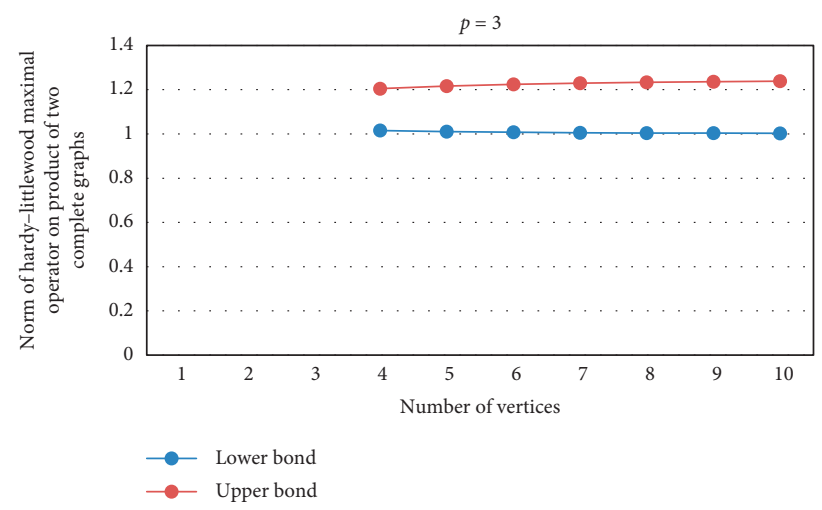

(b)

Figure 2: Estimation for $p=2$ and $p=3$.

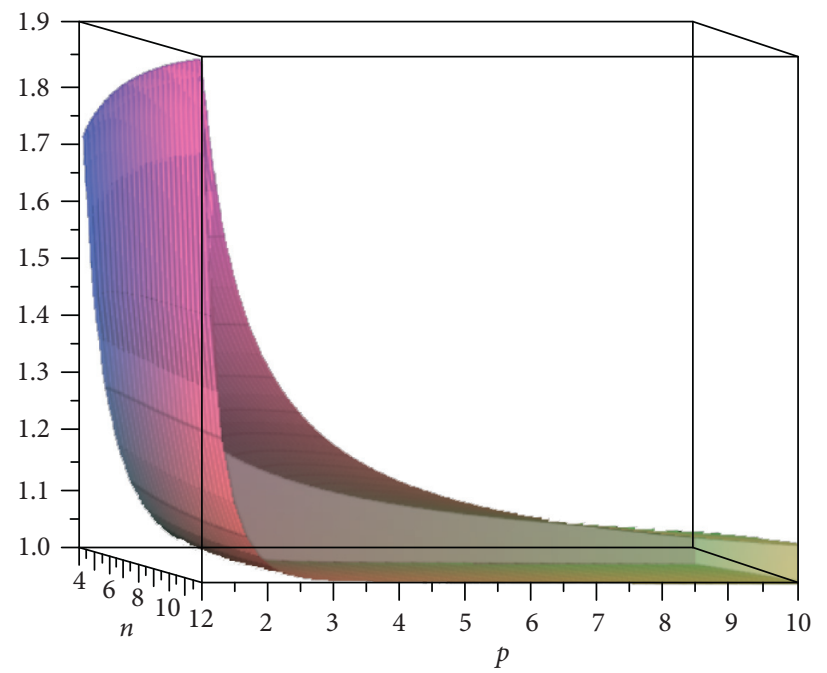

FIgURE 3: 3D view of estimation for $n=4 \ldots 12$ and $p=1 \ldots 10$.

$$
(1+3 \lambda)^{p-1}=\frac{3 \lambda^{p-1}(3 \lambda+1)^{p}+4^{p} \lambda^{p-1}}{9 \lambda^{p}+3} .
$$

In particular, if we take $p=2$, then we get $\lambda=0.246$, and from equation (34), we get $\left\|M_{K_{2} \times K_{2}}^{x}\right\|_{2}=1.151$. If we calculate it from Theorem 1, we get $1.090 \leq\left\|M_{K_{2} \times K_{2}}^{x}\right\|_{2} \leq 1.323$. This shows that the estimation in Theorem 1 is not optimal in general for $1<p<\infty$. Now, in the next theorem, we find the estimates of $G$.

Theorem 2. Let $G$ be the strong product of $m$ graphs and $0<p \leq 1$; then, we have

$$
\begin{aligned}
& \left(\frac{1}{\phi^{p}(1)}+\frac{\left(n_{1} \times n_{2} \times \cdots \times n_{m}\right)-1}{\phi^{p}\left(n_{1} \times n_{2} \times \cdots \times n_{m}\right)}\right)^{(1 / p)} \\
& \quad \leq\left\|M_{G}^{\phi}\right\|_{p} \leq\left(\frac{1}{\phi^{p}(1)}+\frac{\left(n_{1} \times n_{2} \times \cdots \times n_{m}\right)-1}{\phi^{p}\left(2^{m}\right)}\right)^{(1 / p)} .
\end{aligned}
$$

Proof. Lower bound is trivial. For the upper bound, let $\left(u_{1}, u_{2}, \ldots, u_{m}\right) \in V(G)$ and consider the $m$ Dirac delta function $\Gamma_{\left(u_{1}, u_{2}, \ldots, u_{m}\right)}$. Then, we have 


$$
\begin{aligned}
\left\|M_{G}^{\phi} \Gamma_{\left(u_{1}, u_{2}, \ldots, u_{m}\right)}\right\|_{p} & =\left(\left(M_{G}^{\phi} \Gamma_{\left(u_{1}, u_{2}, \ldots, u_{m}\right)}\left(u_{1}, u_{2}, \ldots, u_{m}\right)\right)^{p}+\sum_{v_{1} \neq u_{1}} \sum_{v_{2} \neq u_{2}} \ldots \sum_{v_{m} \neq u_{m}}\left\{M_{G}^{\phi} \Gamma_{\left(u_{1}, u_{2}, \ldots, u_{m}\right)}\left(v_{1}, v_{2}, \ldots, v_{m}\right)\right\}^{p}\right)^{(1 / p)} \\
& =\left(\frac{1}{\phi^{p}(1)}+\sum_{v_{1} \neq u_{1}} \sum_{v_{2} \neq u_{2}} \ldots \sum_{v_{m} \neq u_{m}}\left\{\frac{1}{\phi\left(\left|B_{G_{1}}\right| \times\left|B_{G_{2}}\right| \times \ldots \times\left|B_{G_{m}}\right|\right)} \sum_{w_{1}} \sum_{w_{2}} \ldots \sum_{w_{m}} \Gamma_{\left(u_{1}, u_{2}, \ldots, u_{m}\right)}\left(w_{1}, w_{2}, \ldots, w_{m}\right)\right\}^{p}\right)^{(1 / p)} .
\end{aligned}
$$

As each $G_{i}$ is connected, $\left|B_{G_{i}}\right| \geq 2$ for each $i$ and radius $r \geq 1$. Hence,

$$
\left\|M_{G}^{\phi} \Gamma_{\left(u_{1}, u_{2}, \ldots, u_{m}\right)}\right\|_{p} \leq\left(\frac{1}{\phi^{p}(1)}+\frac{\left(n_{1} \times n_{2} \times \cdots \times n_{m}\right)-1}{\phi^{p}\left(2^{m}\right)}\right)^{(1 / p)} \text {. }
$$

By using Lemma 1, we obtain

$$
\left\|M_{G}^{\phi}\right\|_{p} \leq\left(\frac{1}{\phi^{p}(1)}+\frac{\left(n_{1} \times n_{2} \times \cdots n_{m}\right)-1}{\phi^{p}\left(2^{m}\right)}\right)^{(1 / p)} .
$$

If we take $\phi(x)=x$ and $m=1$, then Theorems 1 and 2, respectively, yields the same results obtained in [9]. This shows that the results presented in this paper are the generalized form of the results in [6].

We have graph for the result of Theorem 2 in Figure 4, where $p=0.5, \phi(x)=x$, and $n_{1} \times n_{2} \times \cdots \times n_{m}$ is from 4 to 10.

Some particular examples to support the result of Theorem 2 are given below.

Example 3. Let $W_{5}$ be a wheel graph on five vertices and consider the strong product $K_{2} \times W_{5}$ of $K_{2}$ with $W_{5}$. Take $\phi(x)=x, f=\Gamma$, and $p=1$. Then, $\left\|M_{K_{2} \times W 5}^{x}\right\|=2.100$.

Let $V\left(K_{2}\right)=\{1,2\}$ and $V\left(W_{5}\right)=\{3,4,5,6,7\}$, where 7 is the central vertex of $W_{5}$. Now, $6 \notin N_{W_{5}}(3)$ and $5 \notin N_{W_{5}}(4)$. Then, the strong product $K_{2} \times W_{5}$ has a vertex set

$$
\begin{aligned}
V\left(K_{2} \times W_{5}\right)= & \{(1,3),(1,4),(1,5),(1,6),(1,7),(2,3), \\
& (2,4),(2,5),(2,6),(2,7)\} .
\end{aligned}
$$

Note that $K_{2} \times W_{5}$ has 37 edges and $d_{(1,7)}\left(K_{2} \times W_{5}\right)=$ $d_{(2,7)}\left(K_{2} \times W_{5}\right)=9$, while all other vertices of this graph have degree 7 . Hence,

$$
M_{K_{2} \times W_{5}}^{x} \Gamma_{(1,3)}= \begin{cases}1, & \text { for }\{(1,3)\} \\ \frac{1}{10}, & \text { for }\{(1,6),(1,7),(2,6),(2,7)\}, \\ \frac{1}{8}, & \text { otherwise, }\end{cases}
$$

with $\left\|M_{K_{2} \times W_{5}}^{x} \Gamma_{(1,3)}\right\|=2.025$. It is easy to see that $\| M_{K_{2} \times W_{5}}^{x}$ $\Gamma_{(1,4)}\|=\| M_{K_{2} \times W_{5}}^{x} \Gamma_{(1,5)}\|=\| M_{K_{2} \times W_{5}}^{x} \Gamma_{(1,6)}\|=\| M_{K_{2} \times W_{5}}^{x} \Gamma_{(2,3)} \|=$ $\left\|M_{K_{2} \times W_{5}}^{x} \Gamma_{(2,4)}\right\|=\left\|M_{K_{2} \times W_{5}}^{x} \Gamma_{(2,5)}\right\|=\left\|M_{K_{2} \times W_{5}}^{x} \Gamma_{(2,6)}\right\|=2.025$.
Also,

$$
M_{K_{2} \times W_{5}}^{x} \Gamma_{(1,7)}= \begin{cases}1, & \text { for }\{(1,7)\} \\ \frac{1}{10}, & \text { for }\{(2,7)\} \\ \frac{1}{8}, & \text { otherwise }\end{cases}
$$

with $\left\|M_{K_{2} \times W_{5}}^{x} \Gamma_{(1,7)}\right\|=2.100,\left\|M_{K_{2} \times W_{5}}^{x} \Gamma_{(2,7)}\right\|=2.100$, and $\left\|M_{K_{2} \times W_{5}}^{x}\right\|=2.100$.

Example 4. Consider the graph used in Example 3. Take $\phi(x)=x^{2}, f=\Gamma$, and $p=1$. Then, $\left\|M_{K_{2} \times W 5}^{x^{2}}\right\|=1.135$. We have

$$
M_{K_{2} \times W_{5}}^{x^{2}} \Gamma_{(1,3)}= \begin{cases}1, & \text { for }\{(1,3)\}, \\ \frac{1}{100}, & \text { for }\{(1,6),(1,7),(2,6),(2,7)\}, \\ \frac{1}{64}, & \text { otherwise, }\end{cases}
$$

with $\left\|M_{K_{2} \times W_{5}}^{x^{2}} \Gamma_{(1,3)}\right\|=1.118$ and $\left\|M_{K_{2} \times W_{5}}^{x^{2}} \Gamma_{(1,4)}\right\|=\| M_{K_{2} \times W_{5}}^{x^{2}}$ $\Gamma_{(1,5)}\|=\| M_{K_{2} \times W_{5}}^{x^{2}} \Gamma_{(1,6)}\|=\| M_{K_{2} \times W_{5}}^{x^{2}} \Gamma_{(2,3)}\|=\| M_{K_{2} \times W_{5}}^{x^{2}} \Gamma_{(2,4)} \|=$ $\left\|M_{K_{2} \times W_{5}}^{x^{2}} \Gamma_{(2,5)}\right\|=\left\|M_{K_{2} \times W_{5}}^{x^{2}} \Gamma_{(2,6)}\right\|=1.118$.

In a similar way, we have

$$
M_{K_{2} \times W_{5}}^{x^{2}} \Gamma_{(1,7)}= \begin{cases}1, & \text { for }\{(1,7)\} \\ \frac{1}{100}, & \text { for }\{(2,7)\} \\ \frac{1}{64}, & \text { otherwise }\end{cases}
$$

with $\left\|M_{K_{2} \times W_{5}}^{x^{2}} \Gamma_{(1,7)}\right\|=1.135$ and $\left\|M_{K_{2} \times W_{5}}^{x^{2}} \Gamma_{(2,7)}\right\|=1.135$. This implies that $\left\|M_{K_{2} \times W_{5}}^{x^{2}}\right\|=1.135$.

Example 5. Let $S_{3}$ be star graph on three vertices and consider the strong product $K_{2} \times K_{2} \times S_{3}$. Take $\phi(x)=x$, $f=\Gamma$, and $p=1$. Then, $\left\|M_{K_{2} \times K_{2} \times S_{3}}^{x}\right\|=2.250$.

Let $V\left(K_{2}\right)=\{1,2\}, V\left(K_{2}\right)=\{3,4\}$, and $V\left(S_{3}\right)=\{5,6,7\}$ with 7 as a central vertex of $S_{3}$. Then, the strong product $K_{2} \times K_{2} \times S_{3}$ is a graph with vertex set 


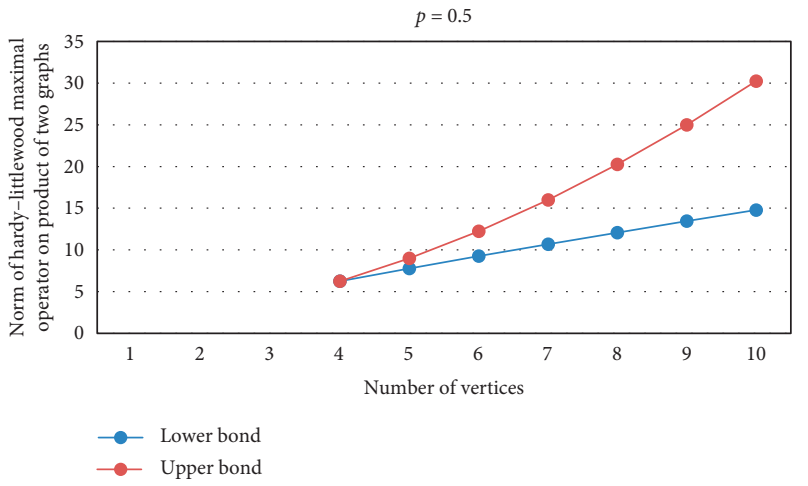

FIGURE 4: Estimation for $p=0.5$.

$V\left(K_{2} \times K_{2} \times S_{3}\right)=\{(1,3,5),(1,3,6),(1,3,7),(1,4,5),(1,4,6),(1,4,7),(2,3,5),(2,3,6),(2,3,7),(2,4,5),(2,4,6),(2,4,7)\}$

Note that there are 50 edges in this graph and $d_{(1,3,7)}\left(K_{2} \times K_{2} \times S_{3}\right)=d_{(1,4,7)}\left(K_{2} \times K_{2} \times S_{3}\right)=d_{(2,3,7)}\left(K_{2} \times\right.$ $\left.K_{2} \times S_{3}\right)=d_{(2,4,7)}\left(K_{2} \times K_{2} \times S_{3}\right)=11$, while all the other vertices of the graph have degree 7 . We have

$$
M_{K_{2} \times K_{2} \times S_{3}}^{x} \Gamma_{(1,3,5)}= \begin{cases}1, & \text { for }\{(1,3,5)\} \\ \frac{1}{8}, & \text { for }\{(1,4,5),(2,3,5),(2,4,5)\} \\ \frac{1}{12}, & \text { otherwise, }\end{cases}
$$

with $\left\|M_{K_{2} \times K_{2} \times S_{3}}^{x} \Gamma_{(1,3,5)}\right\|=2.042$. It is easy to see that $\left\|M_{K_{2} \times K_{2} \times S_{3}}^{x} \Gamma_{(1,3,6)}\right\|=\left\|M_{K_{2} \times K_{2} \times S_{3}}^{x} \Gamma_{(1,4,5)}\right\|=\left\|M_{K_{2} \times K_{2} \times S_{3}}^{x} \Gamma_{(1,4,6)}\right\|$ $=\left\|M_{K_{2} \times K_{2} \times S_{3}}^{x} \Gamma_{(2,3,5)}\right\|=\left\|M_{K_{2} \times K_{2} \times S_{3}}^{x} \Gamma_{(2,3,6)}\right\|=\| M_{K_{2} \times K_{2} \times S_{3}}^{x} \Gamma_{(2,4,5)}$ $\|=\| M_{K_{2} \times K_{2} \times S_{3}}^{x} \Gamma_{(2,4,6)} \|=2.042$.

Also,

$$
M_{K_{2} \times K_{2} \times S_{3}}^{x} \Gamma_{(1,3,7)}= \begin{cases}1, & \text { for }\{(1,3,7)\} \\ \frac{1}{12}, & \text { for }\{(1,4,7),(2,3,7),(2,4,7)\}, \\ \frac{1}{8}, & \text { otherwise, }\end{cases}
$$

with $\left\|M_{K_{2} \times K_{2} \times S_{3}}^{x} \Gamma_{(1,3,7)}\right\|=2.250$. Similarly, $\| M_{K_{2} \times K_{2} \times S_{3}}^{x}$ $\Gamma_{(1,4,7)}\|=\| M_{K_{2} \times K_{2} \times S_{3}}^{x} \Gamma_{(2,3,7)}\|=\| M_{K_{2} \times K_{2} \times S_{3}}^{x} \Gamma_{(2,4,7)} \|=2.250$. This implies that $\left\|M_{K_{2} \times K_{2} \times S_{3}}^{x}\right\|=2.250$.

Example 6. Consider the graph used in example 4, $\phi(x)=1+\ln (x), f=\Gamma$, and $p=1$; then, $\left\|M_{K_{2} \times K_{2} \times S_{3}}^{1+\ln (x)}\right\|=$ 4.459 .

Here, we have

$$
M_{K_{2} \times K_{2} \times S_{3}}^{1+\ln (x)} \Gamma_{(1,3,5)}= \begin{cases}1, & \text { for }\{(1,3,5)\}, \\ \frac{1}{1+\ln (8)}, & \text { for }\{(1,4,5),(2,3,5),(2,4,5)\}, \\ \frac{1}{1+\ln (12)}, & \text { otherwise, }\end{cases}
$$

with $\left\|M_{K_{2} \times K_{2} \times S_{3}}^{1+\ln (x)} \Gamma_{(1,3,5)}\right\|=4.271$. Similarly, $\| M_{K_{2} \times K_{2} \times S_{3}}^{1+\ln (x)}$ $\Gamma_{(1,3,6)}\|=\| M_{K_{2} \times K_{2} \times S_{3}}^{1+\ln (x)} \quad \Gamma_{(1,4,5)}\|=\| M_{K_{2} \times K_{2} \times S_{3}}^{1+\ln (x)} \quad \Gamma_{(1,4,6)} \|=$ $\left\|M_{K_{2} \times K_{2} \times S_{3}}^{1+\ln (x)} \Gamma_{(2,3,5)}\right\|=\left\|M_{K_{2} \times K_{2} \times S_{3}}^{1+\ln (x)} \Gamma_{(2,3,6)}\right\|=\left\|M_{K_{2} \times K_{2} \times S_{3}}^{1+\ln (x)} \Gamma_{(2,4,5)}\right\|$ $=\left\|M_{K_{2} \times K_{2} \times S_{3}}^{1+\ln (x)} \Gamma_{(2,4,6)}\right\|=4.271$.

Now,

$$
M_{K_{2} \times K_{2} \times S_{3}}^{1+\ln (x)} \Gamma_{(1,3,7)}= \begin{cases}1, & \text { for }\{(1,3,7)\}, \\ \frac{1}{1+\ln (12)}, & \text { for }\{(1,4,7),(2,3,7),(2,4,7)\}, \\ \frac{1}{1+\ln (8)}, & \text { otherwise. }\end{cases}
$$

So, $\left\|M_{K_{2} \times K_{2} \times S_{3}}^{1+\ln (x)} \Gamma_{(1,3,7)}\right\|=4.459$. Similarly, $\| M_{K_{2} \times K_{2} \times S_{3}}^{1+\ln (x)}$ $\Gamma_{(1,4,7)}\|=\| M_{K_{2} \times K_{2} \times S_{3}}^{1+\ln (x)} \Gamma_{(2,3,7)}\|=\| M_{K_{2} \times K_{2} \times S_{3}}^{1+\ln (x)} \Gamma_{(2,4,7)} \|=4.459 . \Rightarrow$ $\left\|M_{K_{2} \times K_{2} \times S_{3}}^{1+\ln (x)}\right\|=4.459$.

If we take the same conditions which we used in examples 3-6 in the result of Theorem 2, then we get $1.900 \leq\left\|M_{G_{1} \times G_{2}}^{x}\right\| \leq 3.250,1.090 \leq\left\|M_{G_{1} \times G_{2}}^{x^{2}}\right\| \leq 1.563,1.917 \leq$ $\left\|M_{G_{1} \times G_{2} \times G_{3}}^{x}\right\| \leq 2.375$, and $4.156 \leq\left\|M_{G_{1} \times G_{2} \times G_{3}}^{1+\ln (x)}\right\| \leq 4.572$. This implies that the examples 3-6 verify the result of Theorem 2 .

\section{Conclusion}

In this paper, we have considered the action of generalized maximal operator on $\ell^{p}$ spaces and calculated the quasinorm $\left\|M_{K}^{\phi}\right\|_{p}$ for $0<p \leq 1$. We gave the lower bound and 
upper bound for the quasi-norm $\left\|M_{K}^{\phi}\right\|_{p}$, where $1<p<\infty$. Finally, we have proved that $\left(\left(1 / \phi^{p}(1)\right)+\left(\left(n_{1} \times n_{2} \times \cdots \times\right.\right.\right.$ $\left.\left.\left.n_{m}\right)-1 / \phi^{p}\left(n_{1} \times n_{2} \times \cdots \times n_{m}\right)\right)\right)^{(1 / p)}$ and $\left(\left(1 / \phi^{p}(1)\right)+\left(\left(n_{1} \times\right.\right.\right.$ $\left.\left.\left.n_{2} \times \cdots \times n_{m}\right)-1 / \phi^{p}\left(2^{m}\right)\right)\right)^{(1 / p)}$ are the lower bound and upper bound, respectively.

\section{Data Availability}

No data were used to support this study.

\section{Conflicts of Interest}

The authors declare that they have no conflicts of interest.

\section{Acknowledgments}

The second author and third author thank University of Management and Technology, Lahore, and National Textile University, Faisalabad, for their support.

\section{References}

[1] B. Bolloba's, Modern Graph Theory, Graduate Texts in Mathematics, vol. 184, Springer-Verlag, New York, NY, USA.

[2] A. Bondy and U. S. R. Murty, Graph Theory, Graduate Texts in Mathematics, vol. 244, Springer-Verlag, New York, NY, USA.

[3] R. Hammack, W. Imrich, and S. Klavžar, Handbook of Product Graphs, CRC Press, Boca Raton, FL, USA, 2011.

[4] M. Cowling, S. Meda, and A. G. Setti, "Estimates for functions of the Laplace operator on homogeneous trees," Transactions of the American Mathematical Society, vol. 352, no. 9, pp. 4271-4293, 2000.

[5] C. Gonzalez-Riquelme and J. Madrid, "Sharp P-bounds for maximal operators on finite graphs,” 2020, http://arxiv.org/ abs/2005.03146.

[6] J. Soria and P. Tradacete, "Best constants for the hardy-littlewood maximal operator on finite graphs," Journal of Mathematical Analysis and Applications, vol. 436, no. 2, pp. 661-682, 2016.

[7] J. Soria and P. Tradacete, "Geometric properties of infinite graphs and the hardy-littlewood maximal operator," Journal d'Analyse Mathematique, vol. 1-25, 2016.

[8] C. Capone, D. Cruz-Uribe, A. Fiorenza, and A. Fiorenza, "The fractional maximal operator and fractional integrals on variable $L^{p}$ spaces," Revista Matemática Iberoamericana, vol. 23, no. 3, pp. 743-770, 2007.

[9] I. Ahmad and W. Nazeer, "Optimal couples of rearrangement invariant spaces for generalized maximal operators," Journal of Function Spaces, vol. 2014, Article ID 647123, 5 pages, 2014.

[10] Z. Hussain and S. Talib, "A note on the paper "best constants for the hardy-litllewood maximal operator on finite graphs"” Hacettepe Journal of Mathematics and Statistics, vol. 49, no. 2, pp. 498-504, 2020.

[11] D. Aalto and J. Kinnunen, "The discrete maximal operator in metric spaces," Journal d'Analyse Mathématique, vol. 111, no. 1, pp. 369-390, 2010.

[12] N. Badr and J. M. Martell, "Weighted norm inequalities on graphs," Journal of Geometric Analysis, vol. 22, no. 4, pp. 1173-1210, 2012.

[13] A. Koranyi and M. A. Picardello, "Boundary behaviour of eigenfunctions of the Laplace operator on trees," Annali della Scuola Normale Superiore. Classe di Scienze, vol. 13, no. 3, pp. 389-399, 1986. 